\title{
Acquired pulmonary stenosis due to pleomorphic adenocarcinoma
}

\author{
E JANE FLINT, C WRIGHT, A SINGH \\ From the Departments of Cardiology and Cardiothoracic Surgery, Walsgrave Hospital, Coventry
}

SUMMARY Acquired pulmonary stenosis was diagnosed in a 53 year old woman presenting with the unusual feature of an oral murmur. Mediastinotomy showed extensive malignant tissue surrounding the pulmonary artery, although a mediastinal mass was not evident on chest radiographs. Histological examination confirmed a pleomorphic adenocarcinoma, which has not previously been reported as causing acquired pulmonary stenosis.

The commonest recognised cause of acquired pulmonary stenosis used to be aortic aneurysm. Reviews ${ }^{12}$ and case reports ${ }^{34}$ in recent decades suggest that tumours of the anterior mediastinum, particularly teratomas and Hodgkin's disease, ${ }^{2}$ are now the most frequent culprits leading to compression of the right ventricular outflow tract or main pulmonary artery developing after birth. Whereas invasion of either pulmonary artery by bronchial carcinoma is not uncommon-macroscopic evidence being present in $32 \%$ of surgically resected specimens ${ }^{5}$ - the clinical picture of pulmonary stenosis secondary to neoplasm of the lung has rarely been reported. ${ }^{167}$ There have been reports of two cases of primary chondrosarcoma, ${ }^{67}$ one of primary anaplastic carcinoma of the left main bronchus, ${ }^{1}$ and one of secondary adenocarcinoma. ${ }^{1}$ A metastasis of poorly differentiated bronchogenic carcinoma has caused obstruction of the right ventricular outflow tract, ${ }^{8}$ and pulmonary artery compression has occurred with an intrapericardial mesothelioma. ${ }^{9}$

We report a case of acquired pulmonary stenosis due to a pleomorphic adenocarcinoma, which was probably in its primary site.

\section{Case report}

A 53 year old woman, a telephone operator, was referred to her local ear, nose, and throat clinic in July 1980 with a six week history of hoarseness persisting after an upper respiratory tract infection. An immobile left vocal cord was seen by indirect laryn-

Requests for reprints to Dr E Jane Flint, Department of Cardiology, Walsgrave Hospital, Coventry CV2 2DX. goscopy. Chest radiographs showed a raised left hemidiaphragm but clear lung fields. The symptoms and signs remained unchanged until the beginning of 1982. While sitting in a quiet room she noticed a "saw like" noise, synchronous with her heart beat, when she opened her mouth. During the next few months she had progressive dyspnoea associated with retrosternal chest discomfort on exertion.

On examination in July 1982 she was pale and slim but not unwell and with no palpable lymphadenopathy. She was in sinus rhythm, and her blood pressure was $170 / 95 \mathrm{~mm} \mathrm{Hg}$. In the jugular venous pulse both $A$ and $V$ waves measured $5 \mathrm{~cm}$. There was a harsh ejection systolic murmur, but no associated thrill, which was audible in the aortic and pulmonary areas, neck, and interscapular region and through the open mouth. The chest sounded clear. An electrocardiogram showed only minor lateral $T$ wave changes. Chest radiographs showed no cardiomegaly or mediastinal mass, but in September $1982 \mathrm{a} 1.5 \mathrm{~cm}$ coin lesion appeared in the right midzone on a further chest film, and the patient was referred to this hospital for further investigation. Chest radiographs at this time suggested mild cardiomegaly (Fig. 1a). M mode echocardiography showed paradoxical septal motion and right ventricular dilatation.

Left heart catheterisation confirmed that there was no withdrawal gradient across the aortic valve, and the left ventricular angiogram was normal. At right heart catheterisation a right ventricular pressure of $95 / 10 \mathrm{~mm} \mathrm{Hg}$ and a withdrawal gradient across the pulmonary outflow of $80 \mathrm{~mm} \mathrm{Hg}$ were recorded. The right ventricular angiogram (Fig. 1b) showed irregular compression and an infiltrative obstruction of the left pulmonary artery in particular. The associated 


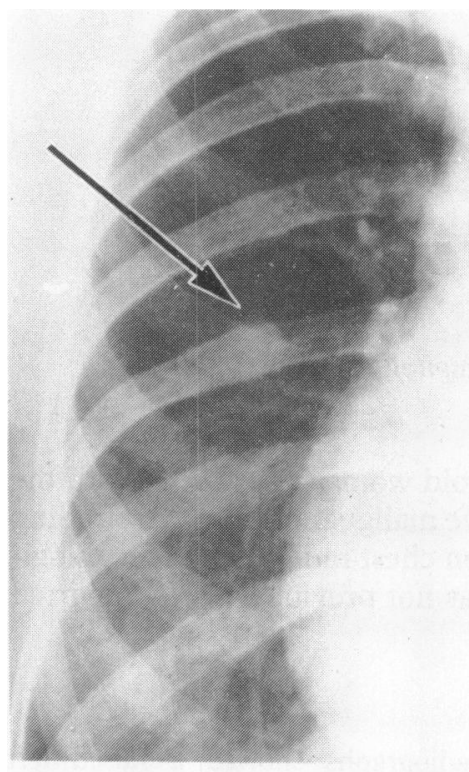

(a)

Fig. 1 (a) Chest radiograph taken in fanuary 1983 showing mild cardiomegaly, coin lesion in right mid-zone, and raised left hemidiaphragm. (b) Right ventricular angiogram showing irregular compression and infiltrative

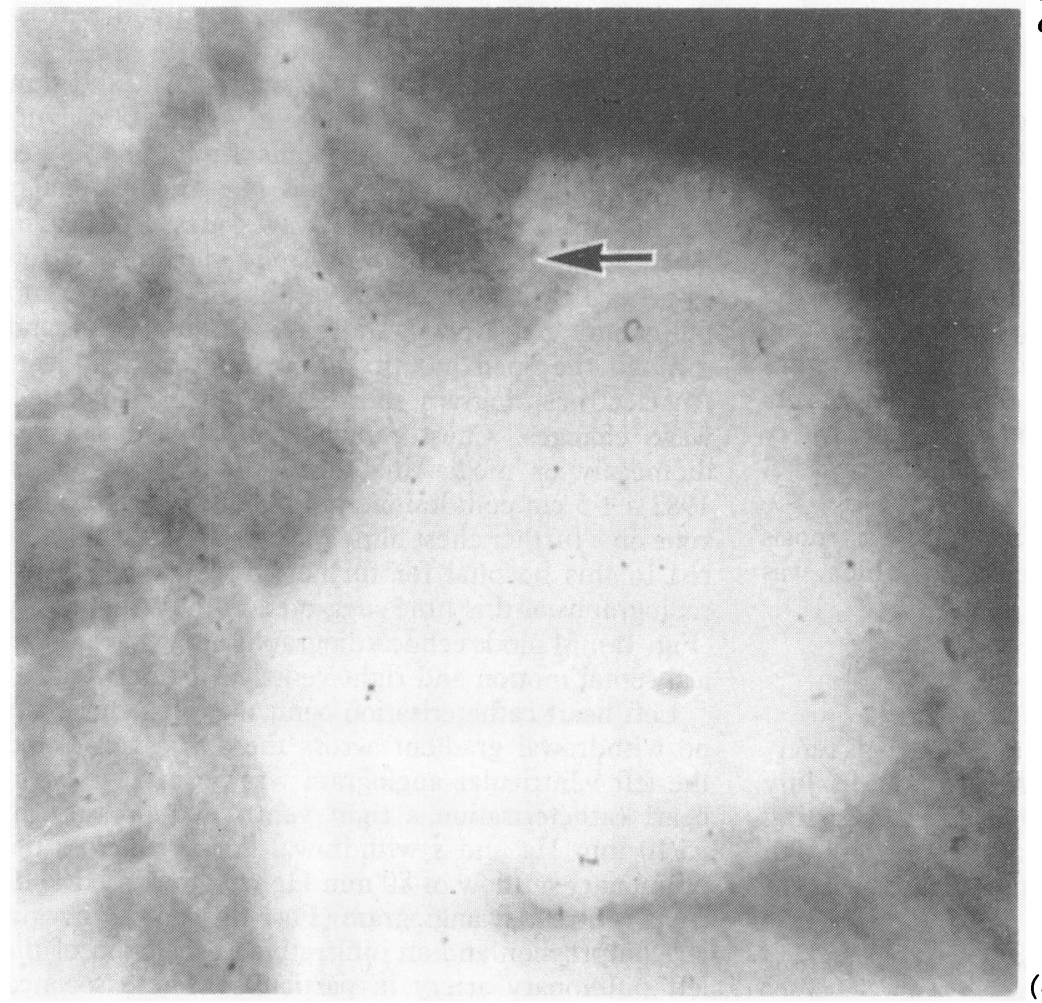
obstruction of the pulmonary artery. 


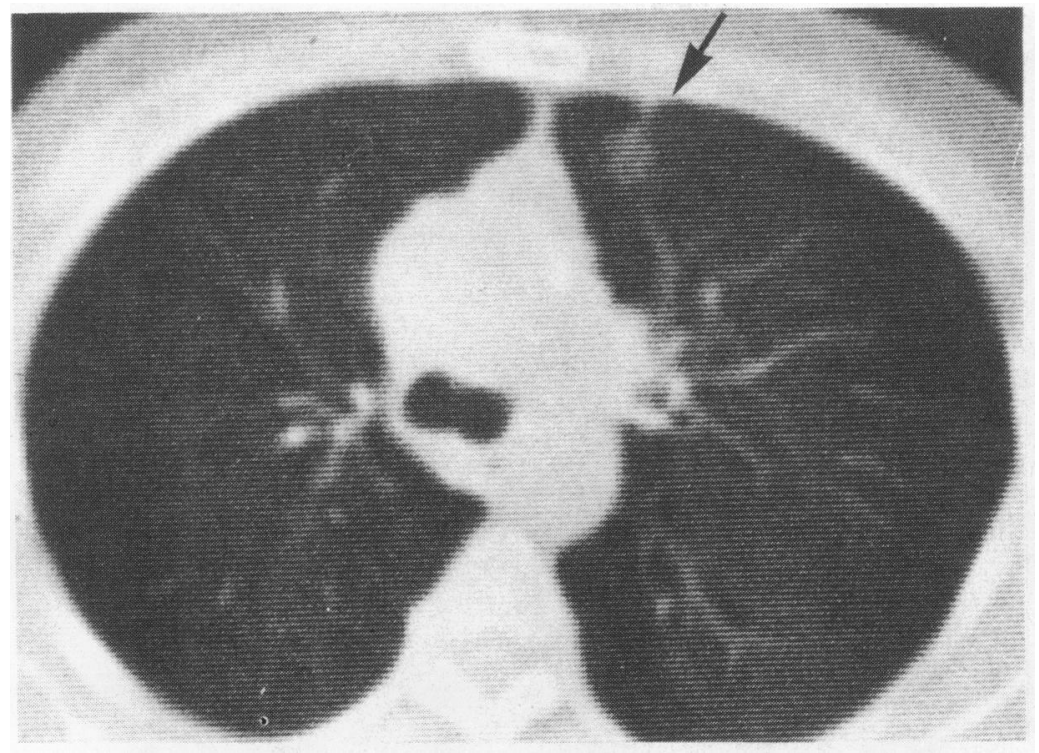

Fig. 1 (c) Computed tomogram of the chest showing irregular distortion of the left hilum, fibrotic change, and nodule in the left upper lobe.

findings of mild tricuspid regurgitation and significant stenosis of the right subclavian vein aroused suspicion of carcinoid syndrome, but this diagnosis was refuted by a negative estimation of urinary 5-hydroxyindole acetic acid. A liver scan was also normal. Computed tomography of the chest (Fig. 1c) showed distortion of the left hilum and fibrotic change in the left upper zone medially. A small nodule suggesting another metastasis was evident in the left upper zone as well as the lesion already seen radiologically in the right mid-zone. The pulmonary artery could not be visualised, but no definite mediastinal mass was identified.

Left anterior mediastinotomy showed a mass of malignant tissue involving the mediastinum and extending into the left upper lobe. The subaortic fossa was full of abnormal tissue, and the pulmonary artery was surrounded by tumour. Histological examination by two independent pathologists of a mediastinal biopsy specimen and a left upper lobe nodule found a poorly differentiated pleomorphic adenocarcinoma (Fig. 2).

Barium investigations failed to show a gastrointestinal primary lesion, and no other primary tumour has so far come to light. The mediastinal tumour may, therefore, be a primary bronchial adenocarcinoma.

In June 1983 the patient was tolerably well, although her activities were limited by grade III dyspnoea. The radiological appearances were unchanged. She was undergoing cytotoxic treatment with cyclophosphamide.

\section{Discussion}

This case of acquired pulmonary stenosis has several interesting points. Firstly, the classical triad of chest pain, dyspnoea, and a pulmonary systolic murmur showed a variation in that the murmur was atypical and not particularly suggestive of pulmonary stenosis. Nevertheless, the combination of a newly acquired atypical bruit and a raised jugular venous pressure aroused suspicion of a mediastinal tumour. The systolic murmur audible through the open mouth was presumably conducted from the infiltrated left pulmonary artery through the tumour mass in the concavity of the aorta to the adjacent left main bronchus posteriorly. The wide radiation of the murmur is probably explained by the extensive solid infiltration of the tumour through the mediastinum.

Secondly, the absence of a definite mediastinal mass visible on chest radiographs is notably unusual and surprising considering the extent of friable tumour at mediastinotomy. Furthermore, computed tomograms did not suggest extensive mediastinal involvement, although a tumour was suggested.

Thirdly, the histology and distribution of the tumour in our patient suggested a mediastinal primary adenocarcinoma with metastases in both lungs. Interestingly, case 10 in Seymour et al's series similarly had no mediastinal mass on chest radiographs but only cardiomegaly. ${ }^{1}$ In their case thoracotomy showed an infiltrating woody mass extending from the sternum to the trachea, which histologically was an adenocarcinoma with a strong fibrous reaction around it. It was thought to be a secondary carcinoma. Adenocarcinoma of the lung is one of the principal histological types to invade arteries. ${ }^{10}$ The primary site of tumours behaving in this way is often indeterminate, ${ }^{5}$ but in our case of pleomorphic adenocar- 


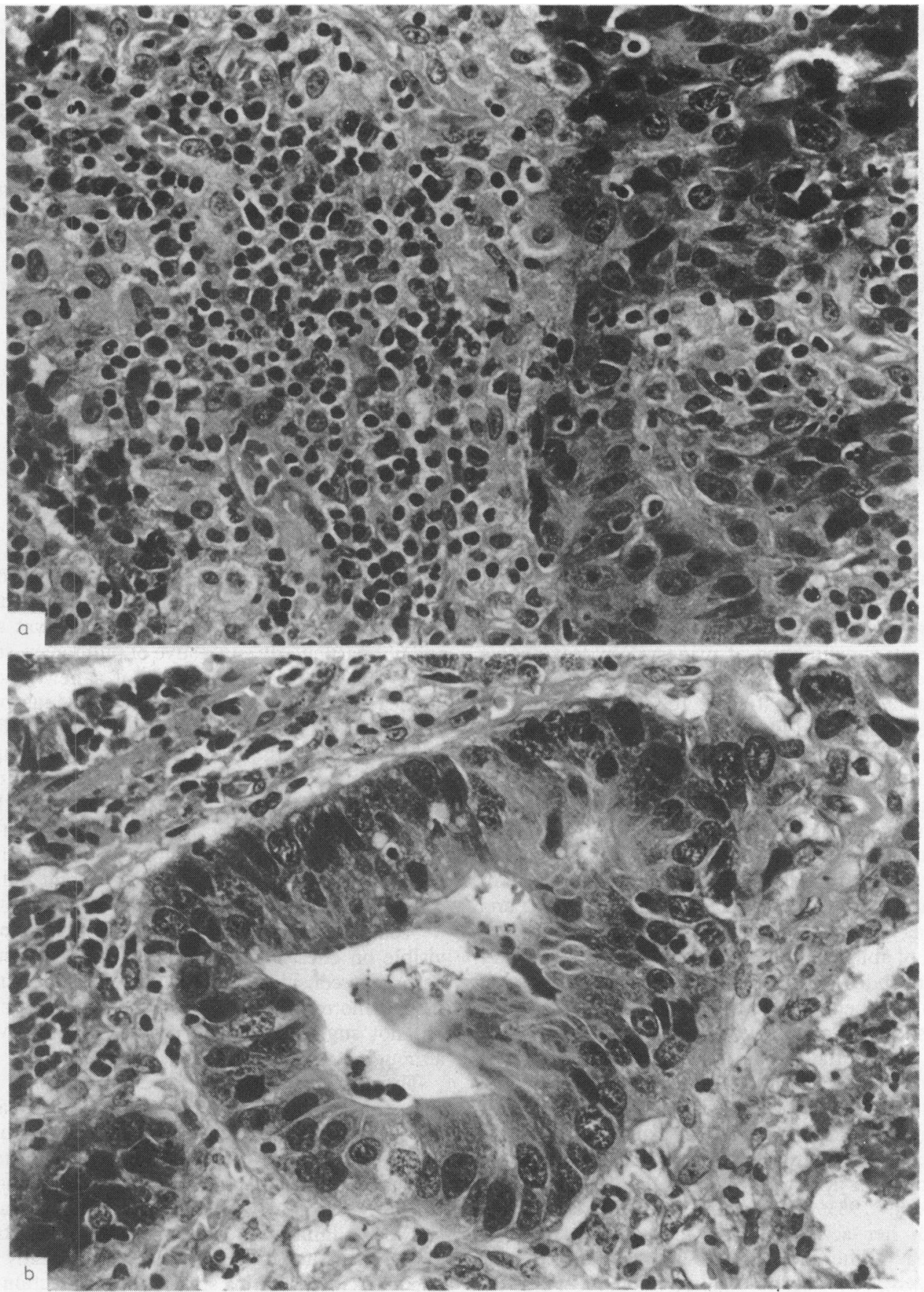

Fig. 2 Histological appearance of (a) mediastinal biopsy specimen (magnification $\times 100 \times 4.5$ ); and (b) left upper lobe nodule (magnification $\times 100 \times 4.5$ ). Both show a pleomorphic adenocarcinoma. 
cinoma it was most probably in the mediastinum.

We thank Dr TT Aye, consultant radiologist, and Dr J Black, consultant pathologist, Walsgrave Hospital, and Dr D Lamb, University of Edinburgh Department of Pathology, for their advice. We also thank Dr J David Hill, Mr JA Dyde, and Dr J Pilcher for their kind permission to report this case.

EJF is in receipt of a Sheldon clinical research fellowship, West Midlands Regional Health Authority.

\section{References}

1 Seymour J, Emanuel R, Pattinson N. Acquired pulmonary stenosis. Br Heart $\mathcal{f}$ 1968; 30: 776-85.

2 Marshall ME, Trump DL. Acquired extrinsic pulmonary stenosis caused by mediastinal tumors. Cancer 1982; 49: 1496-9.

3 Littler WA, Meade JB, Hamilton DI. Acquired pulmo- nary stenosis. Thorax 1970; 25: 465-71.

4 Kindig JR, Tavel ME. Acquired pulmonic stenosis due to a mediastinal seminoma. Chest 1980; 78: 493-5.

5 Pryce DM, Walter JB. The frequency of gross vascular invasion in lung cancer with special reference to arterial invasion. Fournal of Pathology and Bacteriology 1960; 79: 141-6.

6 Greenspan EB. Primary osteoid chondrosarcoma of the lung: report of a case. American foumal of Cancer 1973; 18: 603.

7 Lowell LM, Tuhy JE. Primary chondrosarcoma of the lung. Foumal of Thoracic Surgery 1949; 18: 476-83.

8 McLoughlin MJ. Obstruction of the right ventricular outflow tract due to metastasis. Br $\mathcal{F}$ Radiol 1970; 43: 573-7.

9 Waldhausen JA', Lombardo CR, Morrow AG. Pulmonic stenosis due to compression of the pulmonary artery by an intrapericardial tumour. foumal of Thoracic Surgery 1959; 37: 679-86.

10 Spencer H. Pathology of the lung. Vol. 2. 3rd ed. Oxford: Pergamon Press, 1977: 814 and 848. 